\title{
Interaction of Conical Membrane Inclusions: Effect of Lateral Tension
}

\author{
T. R. Weikl 1), *, M. M. Kozlov ${ }^{1), 2)}$ and W. Helfrich ${ }^{1)}$ \\ 1) Freie Universität Berlin, Fachbereich Physik, Arnimallee 14, 14195 Berlin, Germany \\ 2) Dept. Physiol. Pharmacol., Sackler School of Medicine, Tel-Aviv University, Ramat-Aviv 69978, Israel
}

PACS Numbers: $87.22 \mathrm{Bt}, 87.10+\mathrm{e}, 82.65 \mathrm{Dp}, 34.20-\mathrm{b}$

\begin{abstract}
Considering two rigid conical inclusions embedded in a membrane subject to lateral tension, we study the membrane-mediated interaction between these inclusions that originates from the hatshaped membrane deformations associated with the cones. At non-vanishing lateral tensions, the interaction is found to depend on the orientation of the cones with respect to the membrane plane. The interaction of inclusions of equal orientation is repulsive at all distances between them, while the inclusions of opposite orientation repel each other at small separations, but attract each other at larger ones. Both the repulsive and attractive forces become stronger with increasing lateral tension. This is different from what has been predicted on the basis of the same static model for the case of vanishing lateral tension. Without tension, the inclusions repel each other at all distances independently of their relative orientation. We conclude that lateral tension may induce the aggregation of conical membrane inclusions.
\end{abstract}

\section{INTRODUCTION}

Biological membranes consist of a fluid lipid bilayer with embedded amphiphilic macromolecules such as integral proteins [1]. Integral proteins are expected to be much less flexible than the lipid matrix. In a general sense, any molecule embedded in the membrane and differing in shape or elastic properties from the surrounding lipid molecules can be viewed as an inclusion. The phase behavior of inclusions in the plane of the membrane is determined by interactions between them. If the interaction energy is sufficiently large to compete with translational entropy, it can lead to lateral self-assembly of the inclusions. Attractive forces may result in a lateral aggregation of the inclusions, while repulsion can give rise to a regular array with maximal spacing.

Forces between membrane inclusions can be divided into two classes. The first class consists of the well-known direct interactions, namely electrostatic (for charged inclusions) and van der Waals forces. A second class comprises indirect interactions mediated by some kind of membrane deformation [2] 8]. These interactions are determined by the shapes of the inclusions and the elastic parameters of the inclusions and the lipid bilayer. They can be static or dynamic, in one case being due to equilibrium deformations and in the other to shape fluctuations of the membrane.

Both types of indirect interactions have been theoretically studied for the case of zero lateral tension. The static interactions of inclusions affecting the membrane thickness [3] and of conical deformations affecting the membrane shape [2] have been dealt with. Dynamic interactions were treated for inclusions modifying the local bending moduli [2.4.6.6, including the case of rigid disks [2,5,6].

In the following we consider the static interaction between conical inclusions in the presence of lateral tension. Two sketches of a truncated cone embedded in the membrane are given in Figs. 1 and 2. The cone is assumed to be rigid and to impose a uniform slope on the surrounding membrane which returns asymptotically to the flat state at large distances.

We find repulsive interaction at all values of the lateral tension if the two conical inclusions have equal orientation with respect to the membrane plane. By increasing the lateral tension, the interaction is weakened at larger, but enhanced at smaller inclusion distances. In contrast, for opposite orientations of the inclusions in a membrane with non-vanishing lateral tension the sign of the interaction depends on the distance between the inclusions. At small separations the inclusions repel each other, while at large separations the interaction is attractive. With rising tension the attractive potential well deepens and moves towards smaller distances between the inclusions.

${ }^{*}$ Present address: Max-Planck-Institut für Kolloid- und Grenzflächenforschung, Kantstr. 55, 14513 Teltow, Germany 


\section{SHAPE AND ENERGY OF MEMBRANES WITH CONICAL INCLUSIONS}

We consider a membrane with two embedded conical inclusions. The cross-sections of the inclusions in the midplane of the membrane are circles of radius $a$. The centers of the two circles are separated by the distance $R$ (see Fig. 3).

In the absence of inclusions the membrane is assumed to be flat and to lie in the $x y$-plane of the Cartesian system of coordinates. We describe the membrane equilibrium shape produced by the inclusions by a function $u(x, y)$ which determines the displacement of the membrane from the $x y$-plane in $z$ direction (see Fig. 2).

At the boundaries of the conical inclusions the displacement $u$ is assumed to fulfill the conditions (cf. [2])

$$
\left.u\right|_{r_{i}=a}=h_{i}+a \beta_{i} \cos \phi_{i},\left.\quad \frac{\partial u}{\partial r_{i}}\right|_{r_{i}=a}=\alpha_{i}+\beta_{i} \cos \phi_{i}, \quad i=1,2
$$

where the subscript $i$ takes the values 1 or 2 for the first and the second inclusion, respectively. By $r_{i}$ and $\phi_{i}$ we denote the polar coordinates related to the center of projection $E_{i}$ of the respective inclusion on the $x y$-plane (see Fig. 3). The first equation in (1) describes the boundary of each inclusion as a circle of radius $a$ whose center is at height $h_{i}$ above the $x y$-plane and which is tilted with respect to the $z$ axes by an angle $\beta_{i}$ in the $x$-direction. The second equation in (1) takes into account that due to the conical shape of the inclusion the membrane is attached to the circumference of the tilted circle with a constant angle $\alpha_{i}$. It is assumed in (1) that the contact angle $\alpha_{i}$ and tilt angle $\beta_{i}$ are small, $\alpha_{i} \ll 1, \beta_{i} \ll 1$, so that we set $\tan \alpha_{i}=\alpha_{i}$, $\tan \beta_{i}=\beta_{i}$ and neglect contributions of the order of magnitude of $\beta_{i}^{2}$ determining the deviation of the inclusion projection from the circular shape. At large distances from the inclusions, $r_{i} \gg R$, the membrane remains flat, so that $\nabla u \rightarrow 0$ for $r_{i} \rightarrow \infty$.

The inclusions characterized by small $\alpha_{i}$ and $\beta_{i}$ can produce only a weak deformation of the initially flat membrane, which means that the gradient of the function $u(x, y)$ remains small, $|\nabla u| \ll 1$, everywhere along the membrane. The membrane energy [9] can then be written in the approximate form

$$
G=\int\left(\frac{\kappa}{2}(\Delta u)^{2}+\bar{\kappa} K+\frac{\gamma}{2}(\nabla u)^{2}\right) d^{2} r
$$

where $\kappa$ denotes the bending rigidity, $\gamma$ the lateral tension, $K$ the Gaussian curvature and $\bar{\kappa}$ the modulus of the Gaussian curvature. In our approximation, the Laplacian $\Delta u$ equals the sum of the principal curvatures of the membrane $J$, while $\frac{1}{2}(\nabla u)^{2}$ gives the increase of membrane area per unit projected area due to membrane tilt $\nabla u$. The integration of $(2)$ is performed over the projected area. The membrane shape is determined by the Euler-Lagrange equation following from (2)

$$
\Delta \Delta u=\frac{\gamma}{\kappa} \Delta u
$$

We derive the interaction energy of two conical inclusions in two steps. First, we solve the shape equation (3) accounting for the boundary conditions (1) and the asymptotic boundary condition $\nabla u \rightarrow 0$ for $r_{i} \rightarrow \infty$. Second, inserting the obtained function $u(x, y)$ into (2), we determine the membrane energy. Throughout this calculation we assume the inclusion distance $R$ to be large compared to the radius of inclusion $a$ and retain only the leading terms in $a / R$.

It is important to note that the interaction energy cannot depend on the modulus of Gaussian curvature $\bar{\kappa}$. According to the theorem of Gauss-Bonnet an integral of the Gaussian curvature $K$ over a surface is equal to the negative sum of the line integrals of the geodetical curvature $k_{g}$ over the surface boundaries (apart from a constant that depends only on the genus of the surface). The value of the geodetical curvature at the inclusion boundaries is completely determined by the radius $a$ and contact angles $\alpha_{i}\left(\left|k_{g}\right|=1 / a \cdot \cos \alpha_{i}\right)$ and does not depend on the distance $R$ between the inclusions. Therefore, the integral of the Gaussian curvature $K$ over the membrane must be independent of the distance $R$ and does not contribute to the interaction potential.

For any given distance $R$ between the inclusions the energy has to be minimized with respect to the heights $h_{i}$ and tilt angles $\beta_{i}$. This results in conditions of zero vertical force and zero torque acting on each inclusion. The two conditions are expressed by the equations (see Appendix A)

$$
\int_{0}^{2 \pi}\left[a \frac{\partial}{\partial r_{i}}(\gamma u-\kappa \Delta u)\right]_{r_{i}=a} d \phi_{i}=0
$$




$$
\int_{0}^{2 \pi} \cos \phi_{i}\left[a^{2} \frac{\partial}{\partial r_{i}}(\gamma u-\kappa \Delta u)+\kappa a \Delta u\right]_{r_{i}=a} d \phi_{i}=0,
$$

respectively. The integration in (बi) is performed over the boundary of each inclusion.

\section{INTERACTION IN ABSENCE OF LATERAL TENSION}

We first consider the important limiting case of zero lateral tension, $\gamma=0$. The shape equation (3) then reads

$$
\Delta \Delta u=0 .
$$

To solve this equation satisfying the boundary conditions (1) and the conditions of equilibrium (4) we use the following ansatz. We consider the function $u(x, y)$ describing the shape of the membrane in the form

$$
u=u_{1}\left(r_{1}, \phi_{1}\right)+u_{2}\left(r_{2}, \phi_{2}\right),
$$

where $r_{i}, \phi_{i}$ denote polar coordinates with respect to the center of inclusion $i$. The relationships between the polar coordinates related to the first and the second inclusion are (see Fig. 3)

$$
\begin{aligned}
r_{1} & =\sqrt{R^{2}+r_{2}^{2}-2 R r_{2} \cos \phi_{2}} \\
\cos \phi_{1} & =\frac{r_{2} \cos \phi_{2}-R}{\sqrt{R^{2}+r_{2}^{2}-2 R r_{2} \cos \phi_{2}}}
\end{aligned}
$$

The functions $u_{1}$ and $u_{2}$ in (6) are general solutions of the shape equation (5) in polar coordinates. They are obtained from (B2) derived in Appendix B and have the form:

$$
\begin{aligned}
u_{i}\left(r_{i}, \phi_{i}\right)= & \text { const. }+c_{0}^{(i)} \ln r_{i}+c_{1}^{(i)} r_{i} \cos \phi_{i}+c_{2}^{(i)} r_{i} \ln r_{i} \cos \phi_{i}+c_{3}^{(i)} \frac{\cos \phi_{i}}{r_{i}} \\
& +c_{4}^{(i)} \cos 2 \phi_{i}+c_{5}^{(i)} \frac{\cos 2 \phi_{i}}{r_{i}^{2}}+\ldots+c_{2 n}^{(i)} \frac{\cos n \phi_{i}}{r_{i}^{n-2}}+c_{2 n+1}^{(i)} \frac{\cos n \phi_{i}}{r_{i}^{n}}+\ldots
\end{aligned}
$$

Terms of (B2) proportional to $\sin n \phi$ are omitted in (9) because of the mirror symmetry of the system with respect to the $x z$-plane (see Fig. 3), and terms exhibiting higher than logarithmical divergence for $r_{i} \rightarrow \infty$ are left out since they violate the boundary condition of an asymptotically flat membrane $\boldsymbol{\nabla} u \rightarrow 0$ for $r_{i} \rightarrow \infty$. The only exceptions are $r_{i} \ln r_{i} \cos \phi_{i}$ and $r_{i} \cos \phi_{i}$. From the boundary condition of an asymptotically flat membrane it can be concluded immediately that the coefficient $c_{2}^{(1)}$ must be equal to $-c_{2}^{(2)}$. The sum of the corresponding terms then diverges only logarithmically for $r_{i} \rightarrow \infty$. The terms $r_{i} \cos \phi_{i}$ are proportional to the Cartesian coordinate $x$ and thus describe rotations of the membrane as a whole [10]. Any such rotations must be equal but opposite, i.e. $c_{1}^{(1)}=-c_{1}^{(2)}$, to satisfy the boundary conditions at infinity, so that we can as well drop these terms.

The coefficients $c_{j}^{(i)}$ in (9) are determined from the boundary conditions (11) and equilibrium conditions (4). Consider these conditions at the circumference of inclusion 2. To apply them we have to express the membrane shape (6) in the vicinity of the inclusion. The function $u_{2}$ is simply given by (9) with $i=2$. To present the function $u_{1}$ in a convenient form we take (9) with $i=1$ and insert (7) and (8) into it. In the vicinity of the second inclusion the value of $r_{2}$ is close to the inclusion radius $r_{2} \approx a$. Using the assumption $a \ll R$ and, consequently, $r_{2} \ll R$ we perform a Taylor expansion about the center of the inclusion projection $E_{2}$

$$
\begin{aligned}
\left.u_{1}\right|_{r_{2} \ll R}= & \text { const. }+c_{0}^{(1)}\left(\ln R-\frac{r_{2}}{R} \cos \phi_{2}-\frac{r_{2}^{2}}{2 R^{2}} \cos 2 \phi_{2}\right)-c_{3}^{(1)}\left(\frac{1}{R}+\frac{r_{2}}{R^{2}} \cos \phi_{2}\right) \\
& +c_{2}^{(1)}\left(-R \ln R+(1+\ln R) r_{2} \cos \phi_{2}-\frac{r_{2}^{2}}{2 R}+\frac{r_{2}^{3}}{12 R^{2}}\left(\cos 3 \phi_{2}-3 \cos \phi_{2}\right)\right) \\
& +c_{4}^{(1)}\left(1-\frac{r_{2}^{2}}{R^{2}}\left(1-\cos 2 \phi_{2}\right)\right)+\frac{c_{5}^{(1)}}{R^{2}}-c_{6}^{(1)}\left(\frac{1}{R}+\frac{r_{2}}{R^{2}} \cos \phi_{2}\right)+\frac{c_{8}^{(1)}}{R^{2}}+O\left(\frac{r_{2}^{3}}{R^{3}}\right)
\end{aligned}
$$


The resulting expression for the membrane shape, $u=u_{1}+u_{2}$ is

$$
\left.u\right|_{r_{2} \ll R}=f_{0}^{(2)}\left(r_{2}\right)+f_{1}^{(2)}\left(r_{2}\right) \cos \phi_{2}+f_{2}^{(2)}\left(r_{2}\right) \cos 2 \phi_{2}+f_{3}^{(2)}\left(r_{2}\right) \cos 3 \phi_{2}+\ldots
$$

where

$$
\begin{aligned}
f_{0}^{(2)} & =\text { const. }+c_{0}^{(2)} \ln r_{2}-r_{2}^{2}\left(\frac{c_{2}^{(1)}}{2 R}+\frac{c_{4}^{(1)}}{R^{2}}\right) \\
f_{1}^{(2)} & =c_{2}^{(2)} r_{2} \ln r_{2}+\frac{c_{3}^{(2)}}{r_{2}}+r_{2}\left(-\frac{c_{0}^{(1)}}{R}+c_{2}^{(1)}\left(1+\ln R-\frac{r_{2}^{2}}{4 R^{2}}\right)-\frac{c_{3}^{(1)}+c_{6}^{(1)}}{R^{2}}\right) \\
f_{2}^{(2)} & =c_{4}^{(2)}+\frac{c_{5}^{(2)}}{r_{2}^{2}}+\left(-c_{0}^{(1)}+2 c_{4}^{(1)}\right) \frac{r_{2}^{2}}{2 R^{2}} \\
f_{3}^{(2)} & =\frac{c_{6}^{(2)}}{r_{2}}+\frac{c_{7}^{(2)}}{r_{2}^{3}}+\frac{c_{2}^{(1)} r_{2}^{3}}{12 R^{2}}
\end{aligned}
$$

Inserting (11) into the boundary conditions (11) and equilibrium conditions (4) at the inclusion 2 we obtain a series of equations for the coefficients $c_{j}^{(i)}$.

To account for the boundary and equilibrium conditions at the inclusion 1, we perform the same procedure as described above to obtain identical equations in which the index 2 is replaced by 1 and vice versa.

The equations obtained for the coefficients $c_{j}^{(i)}$ can be solved order by order in the small parameter $a / R$. The solutions are

$$
c_{0}^{(1)}=\alpha_{1} a+O\left(\frac{1}{R^{3}}\right), \quad c_{4}^{(1)}=\frac{\alpha_{2} a^{3}}{R^{2}}+O\left(\frac{1}{R^{3}}\right), \quad c_{5}^{(1)}=-\frac{1}{2} \frac{\alpha_{2} a^{5}}{R^{2}}+O\left(\frac{1}{R^{3}}\right)
$$

and equivalent results for $c_{j}^{(2)}$, the remaining coefficients being of third or higher order in $a / R$.

We are now in a position to compute the energy of the membrane. Omitting the contribution of the integral of the Gaussian curvature, which is independent of the distance $R$ between the inclusions (see above) we obtain from (2)

$$
G(R)=\int \frac{\kappa}{2} J^{2} d^{2} r
$$

where the curvature $J$ is given by

$$
J=\Delta u=-4 c_{4}^{(1)} \frac{\cos 2 \phi_{1}}{r_{1}^{2}}-4 c_{4}^{(2)} \frac{\cos 2 \phi_{2}}{r_{2}^{2}}+\ldots
$$

In the first non-vanishing order in $a / R$, the energy of interaction of the inclusions is

$$
G(R)=4 \pi \kappa\left(\alpha_{1}^{2}+\alpha_{2}^{2}\right) \frac{a^{4}}{R^{4}}+O\left(\frac{1}{R^{5}}\right)
$$

According to (16), the energy is positive and decays monotonically at all values of the contact angles $\alpha_{1}, \alpha_{2}$ and all distances between the inclusions $R$. This means that in the case of zero lateral tension the interaction between the rigid conical inclusions is always repulsive. The result (16) is in agreement with an earlier calculation [2] which in addition predicts a contribution proportional to $\bar{\kappa}$, the modulus of Gaussian curvature. We think that there should be no $\bar{\kappa}$ term (see end of Section II).

\section{INTERACTION IN PRESENCE OF LATERAL TENSION}

We now extend the methods of the previous section to analyze the interactions of inclusions embedded in a membrane subject to non-vanishing lateral tension $\gamma$. The shape equation (3) can be written as 


$$
\Delta \Delta u=\xi^{2} \Delta u,
$$

where $\xi=\sqrt{\gamma / \kappa}$ has the dimension of a reciprocal length. To find a solution of the shape equation satisfying the boundary conditions (17) and equilibrium conditions (4) we use, as in the previous section, ansatz (6) with the functions $u_{i}\left(r_{i}, \phi_{i}\right)$ being general solutions of the shape equation (17) in polar coordinates. These functions are taken from (B3) derived in Appendix B and have the form:

$$
\begin{aligned}
u_{i}= & \text { const. }+c_{0}^{(i)} K_{0}\left(\xi r_{i}\right)+c_{1}^{(i)} r_{i} \cos \phi_{i}+c_{2}^{(i)} K_{1}\left(\xi r_{i}\right) \cos \phi_{i}+c_{3}^{(i)} \frac{\cos \phi_{i}}{r_{i}} \\
& +c_{4}^{(i)} K_{2}\left(\xi r_{i}\right) \cos 2 \phi_{i}+c_{5}^{(i)} \frac{\cos 2 \phi_{i}}{r_{i}^{2}}+\ldots+c_{2 n}^{(i)} K_{n}\left(\xi r_{i}\right) \cos n \phi_{i}+c_{2 n+1}^{(i)} \frac{\cos n \phi_{i}}{r_{i}^{n}}+\ldots
\end{aligned}
$$

The coefficients of all terms of (B3) proportional to $\sin n \phi$ are taken equal to zero because of the symmetry of the system. Also, the coefficients of terms violating the boundary condition of asymptotically vanishing gradient of the displacement, $\nabla u \rightarrow 0$ for $r_{i} \rightarrow \infty$, must be zero. As we do not consider rotations of the system we set $c_{1}^{(i)}=0$. In addition, we omit in (18) the logarithmic term of (B3), as the related change of the area of the membrane would result in an infinite energy of the lateral tension $\gamma$.

Equation (18) transforms into (9) in the limit of vanishing lateral tension, $\gamma \rightarrow 0$ (i.e. $\xi \rightarrow 0$ ). This can be shown by inserting into (18) the approximative expressions of the Bessel functions $K_{n}(x)$ for small arguments $x$

$$
K_{0}(x) \approx-\ln x, \quad K_{1}(x) \approx \frac{1}{x}, \quad K_{n}(x) \approx \frac{(n-1) !}{2}\left(\frac{2}{x}\right)^{n}-\frac{(n-2) !}{2}\left(\frac{2}{x}\right)^{n-2} \text { for } n \geq 2
$$

The coefficients $c_{j}^{(i)}$ in (18) are determined by the boundary conditions (1) and equilibrium conditions (4) in a way similar to that described in the preceding section. For example, we present $u_{1}$ in the vicinity of the inclusion 2 by inserting (7) and (8) and obtain after an expansion in the small parameter $r_{2} / R$

$$
\begin{aligned}
\left.u_{1}\right|_{r_{2} \ll R}= & c_{0}^{(1)}\left(K_{0}(\xi R)+\xi K_{1}(\xi R) r_{2} \cos \phi_{2}+\frac{1}{4} \xi^{2} r_{2}^{2}\left(K_{0}(\xi R)+K_{2}(\xi R) \cos 2 \phi_{2}\right)\right) \\
& +c_{2}^{(1)}\left(-K_{1}(\xi R)-\frac{1}{2} \xi\left(K_{0}(\xi R)+K_{2}(\xi R)\right) r_{2} \cos \phi_{2}\right. \\
& \left.\quad-\frac{1}{8} \xi^{2}\left(K_{1}(\xi R)+K_{3}(\xi R)\right) r_{2}^{2} \cos 2 \phi_{2}-\frac{1}{4} \xi^{2} K_{1}(\xi R) r_{2}^{2}\right) \\
& +c_{4}^{(1)}\left(K_{2}(\xi R)+\frac{1}{2} \xi\left(K_{1}(\xi R)+K_{3}(\xi R)\right) r_{2} \cos \phi_{2}\right. \\
& \left.\quad+\frac{1}{8} \xi^{2}\left(K_{0}(\xi R)+K_{4}(\xi R)\right) r_{2}^{2} \cos 2 \phi_{2}+\frac{1}{4} \xi^{2} K_{2}(\xi R) r_{2}^{2}\right) \\
& -c_{3}^{(1)}\left(\frac{1}{R}+\frac{r_{2}}{R^{2}} \cos \phi_{2}\right)+\frac{c_{5}^{(1)}}{R^{2}}+\ldots
\end{aligned}
$$

We have to stress that in this case the expansions up to the second order in $r_{2} / R$ are sufficient only if $\xi a<1$. For $\xi a \gg 1$, which is equivalent to the condition of strong lateral tension, the series (20) converges too slowly to be approximated by the sum of just a few Taylor terms. This can be seen from the asymptotic expansion of the functions $K_{n}(x)$ which for large arguments are proportional to $\exp (-x) / \sqrt{x}$ irrespectively of $n$.

Inserting the sum (18) with $i=2$ and (20) into the boundary conditions (11) and the equilibrium conditions (4) at inclusion 2, we obtain for $a \ll R$ and $\xi a<1$ a set of linear equations for the coefficients $c_{j}^{(i)}$. Applying the same procedure to satisfy the boundary and equilibrium conditions at the inclusion 1 leads to an analogous set of equations. Solving all these equations for $a \ll R$ and $\xi a<1$ we obtain

$$
\begin{gathered}
c_{0}^{(1)}=-\alpha_{1} a+\ldots, \quad c_{2}^{(1)}=-\frac{1}{2} \alpha_{2} a(\xi a)^{2} K_{1}(\xi R)+\ldots, \\
c_{4}^{(1)}=-\alpha_{2} a(\xi a)^{2} K_{2}(\xi R)+\ldots
\end{gathered}
$$

and corresponding results for $c_{0}^{(2)}, c_{2}^{(2)}$ and $c_{4}^{(2)}$. The coefficients $c_{3}^{(i)}$ and $c_{5}^{(i)}$ are given by the relations 


$$
c_{3}^{(i)}=-\frac{1}{2} c_{2}^{(i)} \xi a^{2} K_{2}(\xi a), \quad c_{5}^{(i)}=-\frac{1}{4} c_{4}^{(i)} \xi a^{3} K_{3}(\xi a) .
$$

The interaction energy $G(R)$ of the inclusions is obtained by integration of $\frac{1}{2} \kappa(\Delta u)^{2}$ over the $x y$-plane. Transforming the area integrals into line integrals over the boundaries of the inclusions as shown in Appendix $\mathrm{C}$ we find the following dominant terms for small $a / R$ and $\xi a<1$

$$
G(R)=2 \pi \kappa \alpha_{1} \alpha_{2}(\xi a)^{2} K_{0}(\xi R)+\pi \kappa\left(\alpha_{1}^{2}+\alpha_{2}^{2}\right)(\xi a)^{4} K_{2}^{2}(\xi R)+\ldots
$$

In the limit of vanishing tension, $\gamma \rightarrow 0$ (i.e. $\xi \rightarrow 0$ ), this expression for the interaction energy coincides with $(16)$ ), as can be seen by expanding the Bessel functions for small arguments according to (19).

The interaction energy (23) depends on the relative orientation of the conical inclusions. If the cones are oriented in the same direction, their contact angles $\alpha_{1}, \alpha_{2}$ have the same sign. In this case the energy of interaction is always positive and decreases with increasing distance $R$. The repulsive potential is illustrated in Fig. 4 for two identical and equally oriented inclusions at different lateral tensions. By increasing the tension the interaction is weakened at large, but enhanced at small inclusion distances.

If the inclusions are oppositely oriented, the contact angles $\alpha_{1}$ and $\alpha_{2}$ have different signs and the energy of interaction behaves non-monotonically, as illustrated in Fig. 5. The energy has a minimum at a finite separation $R^{*}$ of the inclusions. This means that the forces between the inclusions change from repulsive to attractive depending on the distance $R$. For distances shorter than $R^{*}$ the inclusions repel each other, while for separations $R>R^{*}$ the interaction is attractive. With rising lateral tension the separation of zero force, $R^{*}$, moves towards smaller values and the associated potential well deepens (see Fig. 5).

\section{CONCLUSION}

To summarize, we derived an interaction energy between two conical inclusions embedded in a fluid membrane subject to lateral tension. For this purpose, we calculated the equilibrium shape of an almost flat membrane and its bending energy in the presence of inclusions as a function of their distance. In contrast to the case of vanishing tension, this interaction depends on the orientations of the inclusions with respect to the membrane plane. For oppositely oriented inclusions the interaction changes from repulsive to attractive as the separation increases, while equally oriented inclusions repel each other at all distances. This is very different from the case of vanishing lateral tension where the interaction of conical inclusions is always repulsive, independently of relative orientation.

We did not consider in this study the contribution of thermal undulations of the membrane to the interaction between the inclusions [2,5, 6]. In the case of non-vanishing lateral tension this may be partially justified by the fact that the undulations are diminished by the tension. Moreover, others have found for the case of zero tension that the static part of the interaction exceeds the dynamic one for $\kappa\left(\alpha_{1}^{2}+\alpha_{2}^{2}\right)>3 k T$ [2] or $1.5 k T$ [5.,6] where $k$ is Boltzmann's constant and $T$ is temperature. If the tension-induced forces dominate, they should lead to interesting phase behavior of embedded inclusions. For example, the attractive interaction between pairs of oppositely oriented conical inclusions may favor the formation of clusters with a regular structure where inclusions with different signs of the contact angles alternate. For an estimate of the attractive interaction one may use Fig. 5 . With $\kappa=1 \cdot 10^{-19} \mathrm{~J}$ (typical of lipid bilayers), $\alpha=0.5\left(26.8^{\circ}\right)$ and $\xi a=0.4$, the minimum of the interaction energy $G(R)=V(R) \cdot \alpha_{1}^{2} \kappa$ is roughly $-4 \cdot 10^{-21} J(\approx k T$ at room temperature). Because of $\xi=\sqrt{\gamma / \kappa}$ the lateral tension needed to produce $\xi a=0.4$ is given by $\gamma=0.16 \kappa / a^{2}$. For $a=4 \mathrm{~nm}$ and $\kappa=1 \cdot 10^{-19} \mathrm{~J}$, one finds $\gamma=1 \mathrm{mN} / \mathrm{m}$ which is below the known tension of lipid bilayer rupture [1].

While our results are intuitively appealing and may be obtainable more directly, we performed a complete perturbation calculation to make sure that no terms are missed. The boundary and equilibrium conditions for the membrane with conical inclusions are central to our calculations. They resulted in a set of linear equations for the coefficients of two superimposed expansions, one for either inclusion. This method is extendable to larger number of inclusions by using similar sets of boundary conditions. In computer-aided calculation the shape of a membrane could be determined with any desired precision and for any number of inclusions. 


\section{APPENDIX A: DERIVATION OF THE EQUILIBRIUM CONDITIONS}

To derive the equilibrium conditions (4) we study a variation

$$
v(r, \phi, \epsilon)=u(r, \phi)+\epsilon \cdot \delta u(r, \phi)
$$

of the equilibrium membrane displacement $u(r, \phi)$ on a circular ring S: $a \leq r \leq b, 0 \leq \phi \leq 2 \pi$ around a conical inclusion. To simplify the notation we leave out indices of the polar coordinates $r, \phi$. The variation is restricted by the boundary conditions (1) of the inclusion. So

$$
\begin{aligned}
\left.\delta u\right|_{r=a} & =\delta c+\delta \beta a \cos \phi \\
\left.\frac{\partial \delta u}{\partial r}\right|_{r=a} & =\delta \beta \cos \phi
\end{aligned}
$$

where $\epsilon \cdot \delta c$ and $\epsilon \cdot \delta \beta$ denote the changes of the hight of the inclusion center and the tilt angle, respectively. At $r=b$ we set

$$
\left.\delta u\right|_{r=b}=\left.\frac{\partial \delta u}{\partial r}\right|_{r=b}=0 .
$$

Omitting Gaussian curvature the membrane energy (2) can be written as

$$
\begin{aligned}
G & =\int_{S}\left(\frac{\kappa}{2}(\Delta v)^{2}+\frac{\gamma}{2}(\nabla v)^{2}\right) d^{2} r \\
& =\int_{0}^{2 \pi} \int_{a}^{b}\left[\frac{\kappa}{2}\left(\frac{\partial^{2} v}{\partial r^{2}}+\frac{1}{r} \frac{\partial v}{\partial r}+\frac{1}{r^{2}} \frac{\partial^{2} v}{\partial \phi^{2}}\right)^{2}+\frac{\gamma}{2}\left(\left(\frac{\partial v}{\partial r}\right)^{2}+\frac{1}{r^{2}}\left(\frac{\partial v}{\partial \phi}\right)^{2}\right)\right] r d r d \phi \\
& =\int_{0}^{2 \pi} \int_{a}^{b} f\left(v, v_{r}, v_{\phi}, v_{r r}, v_{\phi \phi}, r\right) d r d \phi
\end{aligned}
$$

In equilibrium the energy $G$ is minimal. So

$$
\left.\frac{d G}{d \epsilon}\right|_{\epsilon=0}=\int_{0}^{2 \pi} \int_{a}^{b}\left[\frac{\partial f}{\partial v} \frac{d v}{d \epsilon}+\frac{\partial f}{\partial v_{r}} \frac{d v_{r}}{d \epsilon}+\frac{\partial f}{\partial v_{\phi}} \frac{d v_{\phi}}{d \epsilon}+\frac{\partial f}{\partial v_{r r}} \frac{d v_{r r}}{d \epsilon}+\frac{\partial f}{\partial v_{\phi \phi}} \frac{d v_{\phi \phi}}{d \epsilon}\right]_{\epsilon=0} d r d \phi=0
$$

By partial integrations we obtain

$$
\begin{aligned}
\frac{d G}{d \epsilon}= & \int_{0}^{2 \pi} \int_{a}^{b}\left(\frac{\partial f}{\partial v}-\frac{\partial}{\partial r} \frac{\partial f}{\partial v_{r}}-\frac{\partial}{\partial \phi} \frac{\partial f}{\partial v_{\phi}}+\frac{\partial^{2}}{\partial r^{2}} \frac{\partial f}{\partial v_{r r}}+\frac{\partial^{2}}{\partial \phi^{2}} \frac{\partial f}{\partial v_{\phi \phi}}\right) \frac{d v}{d \epsilon} d r d \phi \\
& +\int_{0}^{2 \pi}\left[\frac{\partial f}{\partial v_{r}} \frac{d v}{d \epsilon}-\left(\frac{\partial}{\partial r} \frac{\partial f}{\partial v_{r r}}\right) \frac{d v}{d \epsilon}+\frac{\partial f}{\partial v_{r r}} \frac{d v_{r}}{d \epsilon}\right]_{a}^{b} d \phi
\end{aligned}
$$

and, inserting $f\left(v, v_{r}, v_{\phi}, v_{r r}, v_{\phi \phi}, r\right)$ as defined in (A5), are led to:

$$
\frac{d G}{d \epsilon}=\int_{0}^{2 \pi} \int_{a}^{b}[\kappa \Delta \Delta v-\gamma \Delta v] \frac{d v}{d \epsilon} r d r d \phi+\int_{0}^{2 \pi}\left[r \frac{\partial}{\partial r}(\gamma v-\kappa \Delta v) \frac{d v}{d \epsilon}+\kappa r \Delta v \frac{d v_{r}}{d \epsilon}\right]_{a}^{b} d \phi
$$

The equilibrium displacement $u(r, \phi)$ fulfills the shape equation (3) of a tense membrane. So the integrand of the area integral in (A8) is zero at $\epsilon=0$. Taking into account $\mathrm{A} 2 \mathrm{~A}$ ) and $\mathrm{A3}$ we conclude

$$
\left.\delta G=\left.\frac{d G}{d \epsilon}\right|_{\epsilon=0}=\int_{0}^{2 \pi}\left[r \frac{\partial}{\partial r}(\gamma u-\kappa \Delta u)(\delta c+\delta \beta a \cos \phi)+\kappa r \Delta u \delta \beta \cos \phi\right)\right]_{r=a} d \phi=0
$$


Since $\delta \beta$ and $\delta c$ are independent of each other we arrive at the equations

$$
\begin{aligned}
& \delta G(\delta c)=\delta c \int_{0}^{2 \pi}\left[a \frac{\partial}{\partial r}(\gamma u-\kappa \Delta u)\right]_{r=a} d \phi=0 \\
& \delta G(\delta \beta)=\delta \beta \int_{0}^{2 \pi} \cos \phi\left[a^{2} \frac{\partial}{\partial r}(\gamma u-\kappa \Delta u)+\kappa a \Delta u\right]_{r=a} d \phi=0
\end{aligned}
$$

which state that the vertical force and the torque, respectively, acting on the inclusion must be zero in equilibrium.

\section{APPENDIX B: GENERAL SOLUTIONS OF THE SHAPE EQUATIONS IN POLAR COORDINATES}

In this appendix we derive the general solution of the shape equation $\Delta \Delta u=\xi^{2} \Delta u$ in polar coordinates. We perform the calculation in two steps. We first look for the solution $J(r, \phi)$ of an intermediate equation $\Delta J=\xi^{2} J$ and then solve the equation $\Delta u=J(r, \phi)$. General solutions of the last equation are also general solutions of the shape equations.

Below we consider separately the case of $\xi=0$, corresponding to the vanishing lateral tension, $\gamma=0$, and the case of non-vanishing $\xi$.

\section{Vanishing lateral tension, $\xi=0$}

In this case the shape equation has the form $\Delta \Delta u=0$. A solution of the intermediate Laplace equation $\Delta J=0$ on a circular ring can be found by the method of separation of variables and reads [12,13]:

$$
J(r, \phi)=a_{0}+b_{0} \ln r+\sum_{n=1}^{\infty}\left(a_{n} \cos n \phi+b_{n} \sin n \phi\right) r^{-n}+\sum_{n=1}^{\infty}\left(c_{n} \cos n \phi+d_{n} \sin n \phi\right) r^{n}
$$

The general solution of the linear inhomogeneous equation $\Delta u=J(r, \phi)$ is the sum of a special solution and the general solution of the homogeneous equation $\Delta u=0$, the latter having the form of (B1). We obtain

$$
\begin{aligned}
& u(r, \phi)=A_{0} r^{2}+B_{0} r^{2}(\ln r-1)+\left(A_{1} \cos \phi+B_{1} \sin \phi\right) r \ln r \\
& \quad+\sum_{n=2}^{\infty}\left(A_{n} \cos n \phi+B_{n} \sin n \phi\right) r^{-n+2}+\sum_{n=1}^{\infty}\left(C_{n} \cos n \phi+D_{n} \sin n \phi\right) r^{n+2} \\
& \quad+\bar{A}_{0}+\bar{B}_{0} \ln r+\sum_{n=1}^{\infty}\left(\bar{A}_{n} \cos n \phi+\bar{B}_{n} \sin n \phi\right) r^{-n}+\sum_{n=1}^{\infty}\left(\bar{C}_{n} \cos n \phi+\bar{D}_{n} \sin n \phi\right) r^{n}
\end{aligned}
$$

The terms with unbarred coefficients belong to the special solution, which can be directly checked by its insertion into $\Delta u=J$. The $A_{0}$-term of (B2) corresponds to the $a_{0}$-term of (B1) etc. The terms with barred coefficients give the general solution of $\Delta u=0$ in analogy to (B1).

\section{Non-vanishing lateral tension, $\xi \neq 0$}

By applying the method of separation of variables described in [12,13 also to the case of non-vanishing lateral tension we find the following general solution of the intermediate equation $\Delta J=\xi^{2} J$

$$
J(r, \phi)=a_{0} K_{0}(\xi r)+\sum_{n=1}^{\infty}\left(a_{n} \cos n \phi+b_{n} \sin n \phi\right) K_{n}(\xi r)+\sum_{n=1}^{\infty}\left(c_{n} \cos n \phi+d_{n} \sin n \phi\right) I_{n}(\xi r)
$$

where $I_{n}$ and $K_{n}$ denote modified Bessel functions. A general solution of the equation $\Delta u=J(r, \phi)$ again consists of the sum of a special solution and the general solution (B1) of the Laplace equation $\Delta u=0$. It can be written in the form 


$$
\begin{aligned}
& u(r, \phi)=A_{0} K_{0}(\xi r)+B_{0} I_{0}(\xi R)+\bar{A}_{0}+\bar{B}_{0} \ln r \\
& \quad+\sum_{n=1}^{\infty}\left(A_{n} \cos n \phi+B_{n} \sin n \phi\right) K_{n}(\xi r)+\sum_{n=1}^{\infty}\left(C_{n} \cos n \phi+D_{n} \sin n \phi\right) I_{n}(\xi r) \\
& \quad+\sum_{n=1}^{\infty}\left(\bar{A}_{n} \cos n \phi+\bar{B}_{n} \sin n \phi\right) r^{-n}+\sum_{n=1}^{\infty}\left(\bar{C}_{n} \cos n \phi+\bar{D}_{n} \sin n \phi\right) r^{n}
\end{aligned}
$$

taking into account that $K_{0}(\xi r), K_{n}(\xi r) \cos n \phi, I_{0}(\xi r), I_{n}(\xi r) \cos n \phi$ and the corresponding terms containing $\sin n \phi$ are eigenfunctions of the Laplace operator.

\section{APPENDIX C: REDUCING AREA INTEGRALS TO LINE INTEGRALS IN THE CALCULATION OF THE MEMBRANE ENERGY}

In the calculation of the energy of the tense membrane

$$
G=\int\left[\frac{\kappa}{2}(\Delta u)^{2}+\frac{\gamma}{2}(\nabla u)^{2}\right] d^{2} r
$$

we encounter, due to our ansatz (18), integrals of the form

$$
I(f, g)=\int_{\boldsymbol{R}^{2} / E_{1} \cup E_{2}}\left[\frac{\kappa}{2} \Delta f \Delta g+\frac{\gamma}{2} \nabla f \cdot \nabla g\right] d^{2} r
$$

where either $f$ obeys $\Delta f=\gamma / \kappa \cdot f$, which is true for the terms of (18) containing a Bessel function, or $g$ is a solution of $\Delta g=0$, or both (see Appendix B). $E_{i}$ denotes the projection of the inclusion $i$ into the $x y$-plane (see Fig. 3). Applying a theorem of Green we may write

$$
\int_{\boldsymbol{R}^{2} / E_{1} \cup E_{2}}(\nabla f \cdot \nabla g) d^{2} r=-\int_{\boldsymbol{R}^{2} / E_{1} \cup E_{2}} f \Delta g d^{2} r-\left.\int_{0}^{2 \pi} f \frac{\partial g}{\partial r_{1}}\right|_{r_{1}=a} a d \phi_{1}-\left.\int_{0}^{2 \pi} f \frac{\partial g}{\partial r_{2}}\right|_{r_{2}=a} a d \phi_{2}
$$

if $r_{i} f\left(\partial g / \partial r_{i}\right)$ goes to zero for $r_{i} \rightarrow \infty$. Since

$$
\int_{\boldsymbol{R}^{2} / E_{1} \cup E_{2}}\left[\frac{\kappa}{2} \Delta f \Delta g-\frac{\gamma}{2} f \Delta g\right] d^{2} r=0
$$

for $\Delta f=\gamma / \kappa \cdot f$ or $\Delta g=0$, we find

$$
I(f, g)=-\left.\frac{\gamma}{2} \int_{0}^{2 \pi} f \frac{\partial g}{\partial r_{1}}\right|_{r_{1}=a} a d \phi_{1}-\left.\frac{\gamma}{2} \int_{0}^{2 \pi} f \frac{\partial g}{\partial r_{2}}\right|_{r_{2}=a} a d \phi_{2} .
$$

[1] B. Alberts et al., Molecular Biology of the Cell (Garland, New York, 1983)

[2] M. Goulian, R. Bruinsma and P. Pincus, Europhys. Lett., 22 (2), 145 (1993) and Erratum in Europhys. Lett., 23 (2), 155 (1993)

[3] N. Dan, A. Berman, P. Pincus and S.A. Safran, J. Phys. II France 4, 1713 (1994)

[4] R. Netz and P. Pincus, Phys. Rev. E 52, 4114 (1995)

[5] R. Golestanian, M. Goulian and M. Kardar, Europhys. Lett. 33 (3), 241 (1996)

[6] J.-M. Park and T.C. Lubensky, J. Phys. I France 6, 1217 (1996)

[7] R. Netz, J. Phys. I France 7, 833 (1997) 
[8] For recent reviews see: M. Goulian, Current Opinion in Colloid \& Interface Science, 1:358 (1996), R. Bruinsma and P. Pincus, Current Opinion in Solid State \& Materials Science, 1:401 (1996)

[9] W. Helfrich, Z. Naturforsch. 28c, 693 (1973)

[10] The shape $u$ of a tensionless membrane with a single inclusion for example is just $u=\alpha a \ln r$. Enforcing a tilt of the inclusion by an angle $\beta$ results in the shape $u=\alpha a \ln r+\beta r \cos \phi$, i.e. a tilt of the whole membrane by $\beta$.

[11] E. Evans and D. Needham, J. Phys. Chem. 91, 4219 (1987)

[12] J. D. Jackson, Classical Electrodynamics, 2nd ed. (Wiley, New York, 1975)

[13] M.A. Pinsky, Partial Differential Equations and Boundary-Value Problems with Applications (McGraw-Hill, New York, 1991)

\section{Figure Captions:}

Figure 1:

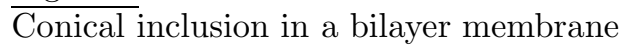

Figure 2:

Idealization of a conical inclusion as rigid disc of height $h_{i}$ and tilt angle $\beta_{i}$ making a uniform contact angle $\alpha_{i}$ with the surrounding membrane. The cross section contains the axis of the cone.

Figure 3:

$\overline{x y \text {-plane }}$ with inclusion projections $E_{1}$ and $E_{2}$

Figure 4:

Dimensionless interaction potential $V(R)=G(R) /\left(\alpha_{1}^{2} \kappa\right)$ of two equally oriented inclusions

$\left(\alpha_{1}=\alpha_{2}\right)$ as a function of the dimensionless distance $R / a$ for $\xi a=0.05,0.1,0.2$

Figure 5:

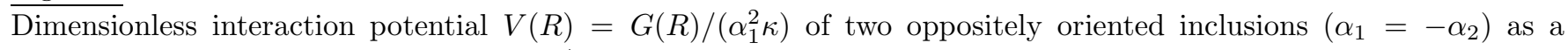
function of the dimensionless distance $R / a$. The potential well deepens with increasing $\xi a=0.1,0.2,0.3,0.4,0.5$. Note that $R / a=2$ means two discs in contact. Near this value the results can only be regarded as estimates. 


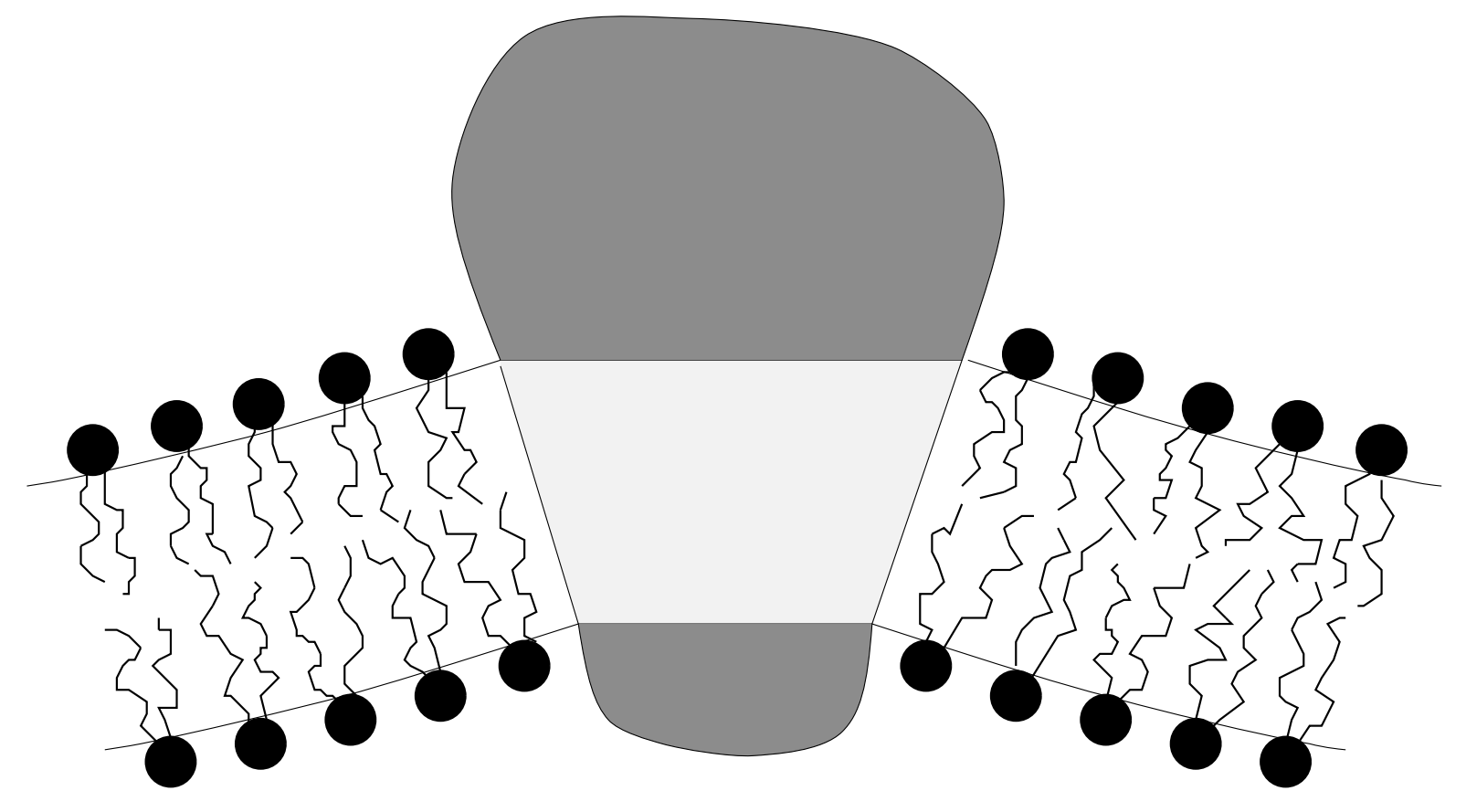




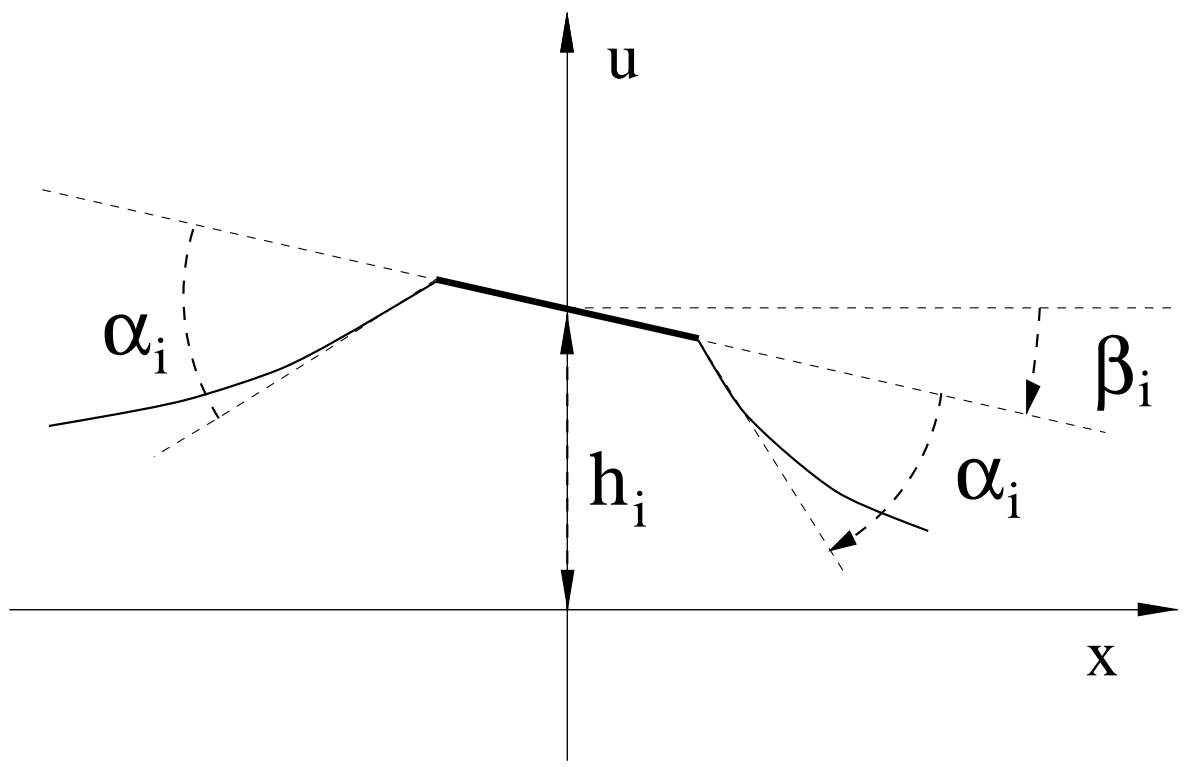




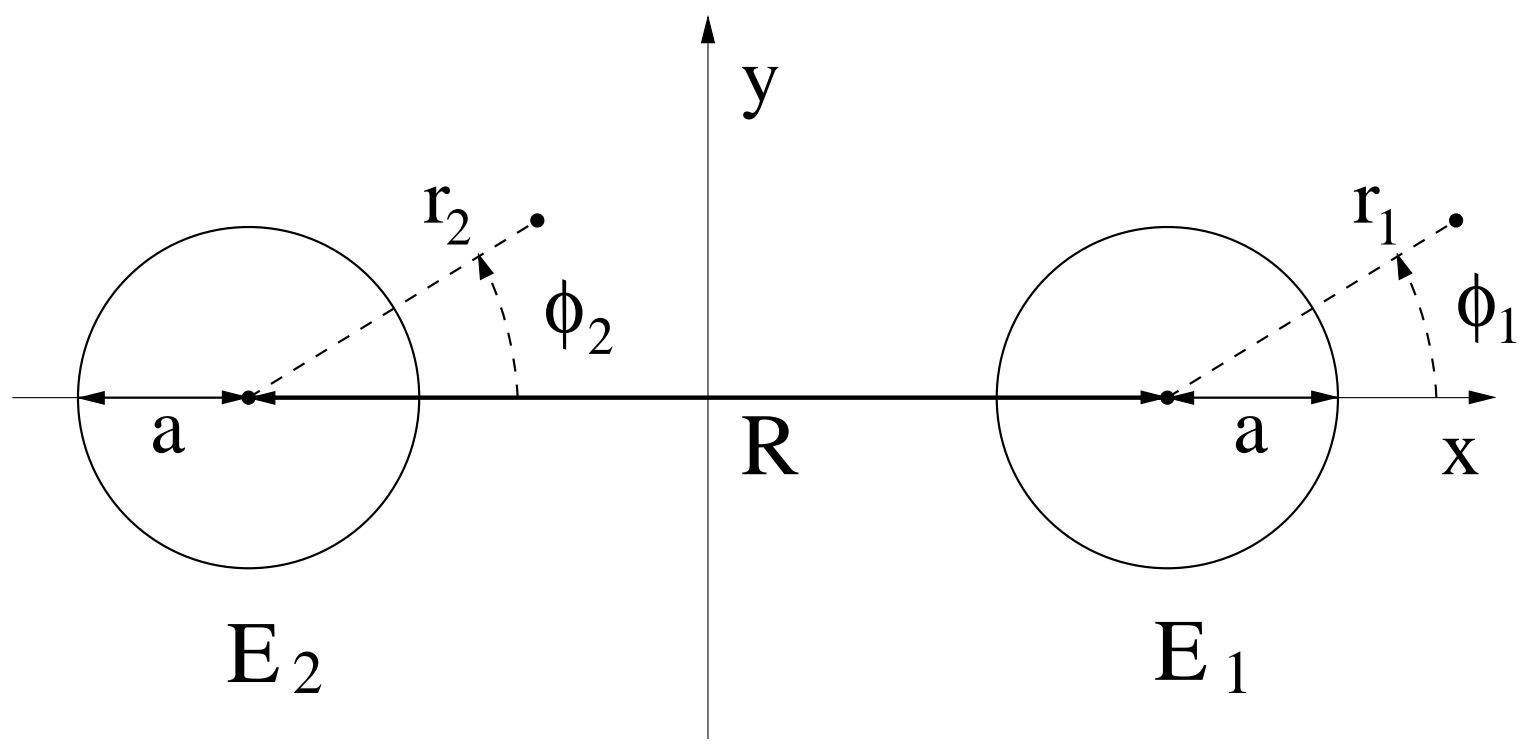




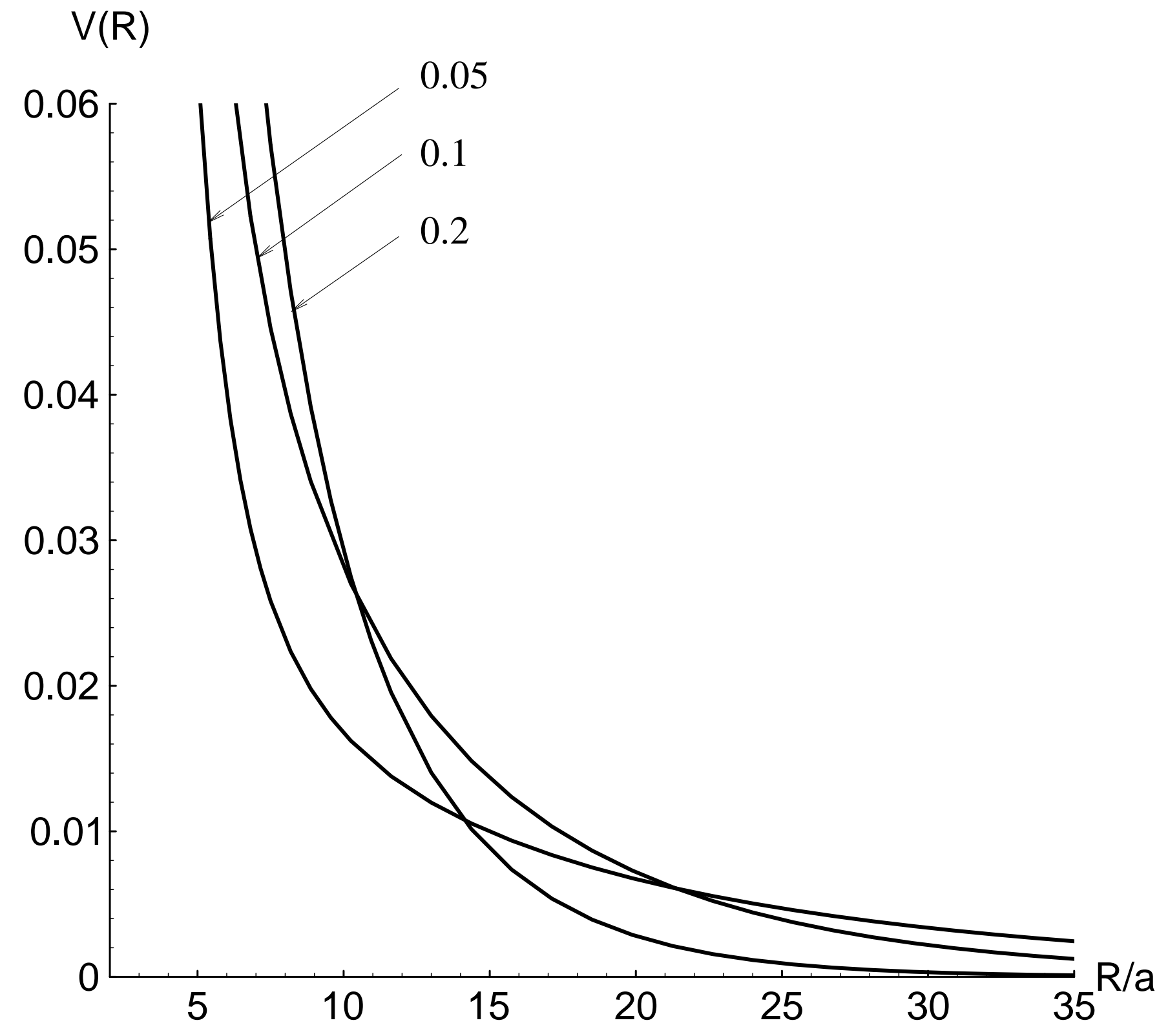




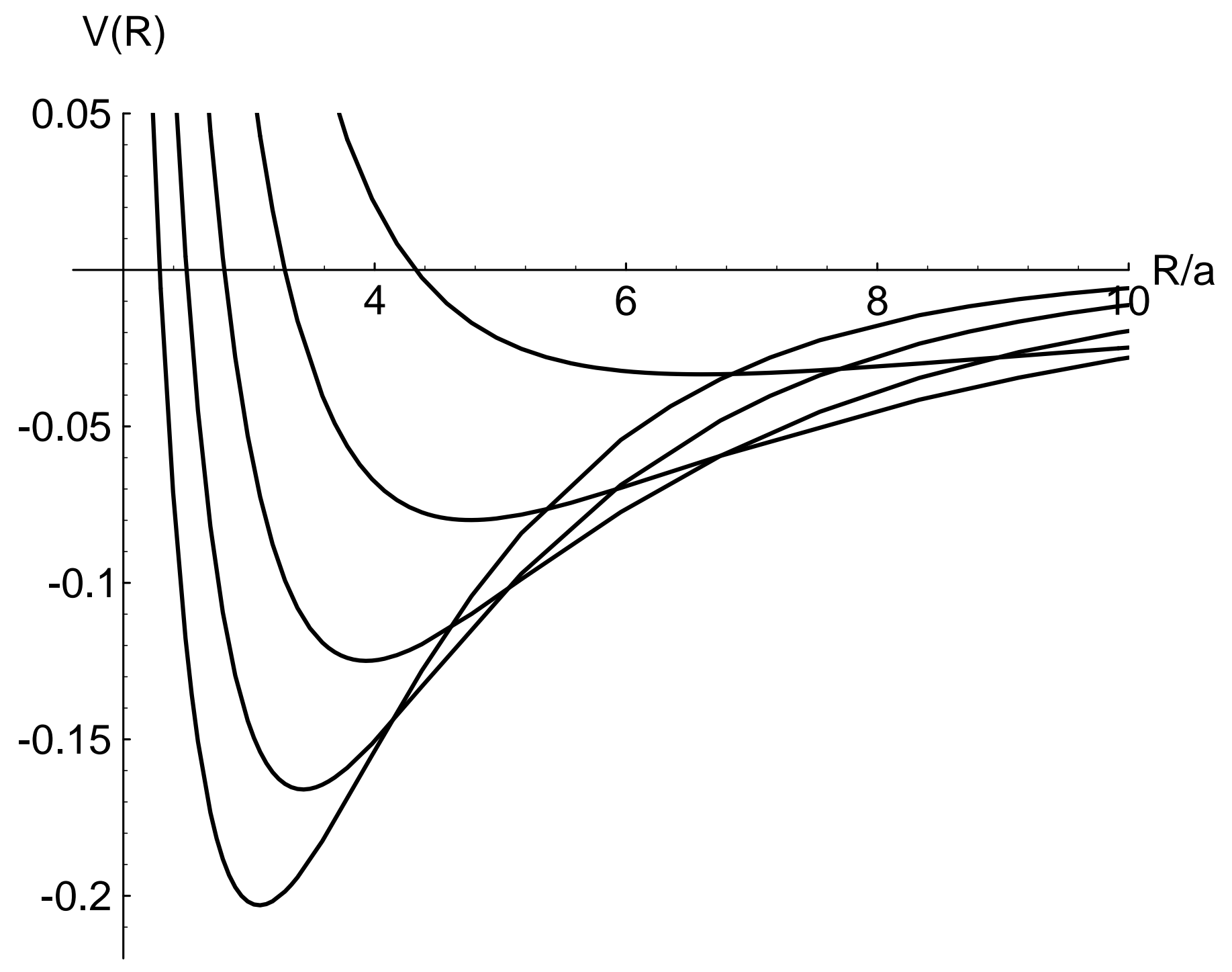

